\title{
From Random Matrix Theory to Statistical Mechanics - Anyon Gas
}

\author{
Daniel Alonso ${ }^{1,2}$ and Sudhir R. Jain ${ }^{2,3}$ \\ (1) Department of Fundamental and Experimental Physics, \\ University of La Laguna, La Laguna 38204 \\ Tenerife, Canary Islands, Spain \\ (2) Faculté des Sciences and \\ Center for Nonlinear Phenomena and Complex Systems, \\ Université Libre de Bruxelles, Campus Plaine C.P. 231, \\ Boulevard du Triomphe, 1050 Bruxelles, Belgium \\ (3) Theoretical Physics Division, Bhabha Atomic Research Centre, \\ Bombay 400085, India
}

\begin{abstract}
Motivated by numerical experiments and studies of quantum systems which are classically chaotic, we take a Random Matrix description of a Hardsphere gas to Statistical Mechanical description. We apply this to Anyon gas and obtain a formal expression for the momentum distribution. Various limiting situations are discussed and are found in agreement with the well-known results on Hard-sphere gas in low-density regime.
\end{abstract}

PACS numbers:05.30.-d 05.45.+b 
Random Matrix Theory and Statistical Mechanics are employed for the description of many-body systems like nuclei, metallic clusters and so on. During the last decade or so, it has become increasingly clear that spectral fluctuations of simple quantum systems [1] whose classical counterpart are chaotic, as well as the eigenfunctions, have features [2] in striking agreement with random matrix theory (RMT). Originating from a conjecture of Berry [3] regarding the eigenfunctions as a random superposition of plane waves, it was shown that a contact with statistical mechanics can be realised [四]. In this Letter, we show that this connection is indeed a way to relate RMT and Statistical Mechanics.

It is well-known that statistical mechanics in two dimensions is at the core of several phenomena of great interest such as the fractional Hall effect, high- $T_{c}$ superconductivity, and others [5].6]. Unlike in three dimensions the analysis here is plagued with difficulties stemming from an unusual, non-local exchange interaction potential. As a result identical particles in two dimensions exhibit statistics interpolating between the Fermi-Dirac and Bose-Einstein distributions, generically these are termed as Anyons [7]. In this Letter, we address this fundamental problem in two dimensions and cast it systematically employing an ansatz for the eigenfunctions consistent with the connection mentioned above, and incorporate the Braid group governing exchange symmetries. We show a way to arrive at the momentum distribution upto $O\left(\hbar^{2}\right)$ and express it in terms of quantities involving the counting problem in Braid group. Statistical mechanics of Anyons has been studied in the past [8] and it is very interesting to see that the second virial coefficient obtained then and now, give rise to a Sum Rule. However, in marked distinction with previous works, calculation of higher-order virial coefficients is also possible here - a facet which makes the present approach a novel alternative. To this end, we argue that the third virial coefficient has the form in agreement with some recent results. We re-iterate that the aim of this Letter is to arrive at quantum statistical mechanics without having to make a hypothesis about thermal bath, and that the present approach is guided by random matrix theory.

The fundamental hypotheses of RMT are (a) the hamiltonian of a given system belongs to an ensemble of hamiltonians, and (b) real quantum mechanics is enough for description of 
physical systems if time-reversal symmetry is taken into account appropriately [9]. It is the latter of the two that leads to exactly three universality classes in the RMT - Orthogonal (even spin, time-reversal invariant, OE) Unitary (time-reversal non-invariant, UE) and Symplectic Ensembles (odd spin, Time-Reversal Invariant, SE) [10]. Owing to the spin-statistics connection in two dimensions [11], we know that there is fractional statistics and fractional spin. In the context of symmetries, in two dimensions, parity $(\mathrm{P})$ and time-reversal $(\mathrm{T})$ both are broken.

Let us consider a system of $N$ hard spheres ('discs' in two dimensions), each of radius $a$, enclosed in a box of edge-length $L+2 a$. Centres of two hard spheres $\vec{x}_{i}$ and $\vec{x}_{j}$ are such that $\left|\vec{x}_{i}-\vec{x}_{j}\right| \geq 2 a$. The canonical pair of coordinates describing these particles are $(\vec{X}, \vec{P})$ where $\vec{X}=\left(\vec{x}_{1}, \vec{x}_{2}, \cdots, \vec{x}_{N}\right), \vec{P}=\left(\vec{p}_{1}, \vec{p}_{2}, \cdots, \vec{p}_{N}\right)$. Energy eigenfunctions, $\psi_{\alpha}(\vec{X})$ corresponding to eigenvalue $E_{\alpha}$ vanish on the boundary of the enclosure. A typical eigenfunction is irregular, with a Gaussian amplitude distribution and the spatial correlation function of the same is consistent with the conjecture of Berry which allows us to represent this eigenfunction as a superposition [ [] :

$$
\psi_{\alpha}(\vec{X})=N_{\alpha} \int d^{d N} \vec{P} A_{\alpha}(\vec{P}) \delta\left(P^{2}-2 m E_{\alpha}\right) e^{\frac{i}{\hbar} \vec{X} \cdot \vec{P}}
$$

with $N_{\alpha}$ given by the normalization constant, and $A_{\alpha}^{\prime} s$ satisfying the two-point correlation function

$$
\left\langle A_{\alpha}^{*}(\vec{P}) A_{\gamma}\left(\vec{P}^{\prime}\right)\right\rangle_{M E}=\delta_{\alpha \gamma} \frac{\delta^{d N}\left(\vec{P}-\vec{P}^{\prime}\right)}{\delta\left(\vec{P}^{2}-\vec{P}^{\prime 2}\right)}
$$

$d$ denotes the number of coordinate-space dimensions. The average in (2) is a matrixensemble (ME) average which originates from the fact that the hamiltonian, $H$ of the system belongs to an ensemble of matrices satisfying associative division algebra [10,12 in consistency with quantum mechanics. The eigenstate ensemble (EE) used in [1 is nothing but a consequence of underlying matrix ensemble in RMT, the eigenfunctions then satisfy all the properties numerically observed and analytically represented in (11), (2) [13]. The correlation functions (2) decide whether time-reversal symmetry is preserved $\left(A_{\alpha}^{*}(\vec{P})=A_{\alpha}(-\vec{P})\right)$ 
or broken $\left(A_{\alpha}^{*}(\vec{P}) \neq A_{\alpha}(-\vec{P})\right)$, accordingly the corresponding matrix ensemble belongs to $\mathrm{OE}$ or UE respectively. As noted in 四, the higher-order even-point correlation functions factorize and the odd-ones vanish. A very important aspect of the ansatz (1), (2) is that the Wigner function corresponding to $\psi_{\alpha}(\vec{X})$ is microcanonical, or, is proportional to $\delta\left(H-E_{\alpha}\right)$ which, in a sense, incorporates ergodicity. We note here that, starting from an ansatz very similar to the one above, it is possible to obtain the quantum transport equation [14] where it is important to relate a given quantum state with the admissible energy surface in phase space; thus the above ansatz is in conceptual agreement with the ergodic aspect of manybody system. Moreover, this choice fixes the Thomas-Fermi density of states naturally. It now becomes important to emphasize that we must restrict ourselves to dilute gas of hardspheres and also assume that the size of sphere is much lesser than the thermal de Broglie wavelength. Thus, the ansatz establishes, in fact, a link between RMT and statistical mechanics. We now incorporate the case of two dimensions which otherwise presents enormous difficulties.

In two dimensions, the solutions of the Schrödinger equation, $\psi\left(\vec{x}_{1}, \vec{x}_{2}, \cdots, \vec{x}_{N}\right)$, under an exchange of two coordinates of particles satisfies

$$
\begin{array}{r}
\psi\left(\vec{x}_{1}, \cdots, \vec{x}_{i}, \cdots, \vec{x}_{j}, \cdots, \vec{x}_{N}\right) \\
=e^{i \pi \nu} \psi\left(\vec{x}_{1}, \cdots, \vec{x}_{j}, \cdots, \vec{x}_{i}, \cdots, \vec{x}_{N}\right)
\end{array}
$$

where $\nu$ is arbitrary and defines statistics. For $\nu=0$ and $\nu=1$, with (2), one gets the BoseEinstein and Fermi-Dirac distributions. This non-trivial phase and the resulting boundary condition arises from the fact that the effective configuration space, $M_{N}^{2}$ has a fundamental group, $\pi_{1}\left(M_{N}^{2}\right)=B_{N}$ [15], the Braid group of $N$ objects which is an infinite, non-abelian group. $B_{N}$ is generated by $(N-1)$ elementary moves $\sigma_{1}, \cdots, \sigma_{N-1}$ satisfying the Artin relations,

$$
\begin{array}{r}
\sigma_{i} \sigma_{i+1} \sigma_{i}=\sigma_{i+1} \sigma_{i} \sigma_{i+1}(i=1,2, \cdots, N-2) \\
\sigma_{j} \sigma_{i}=\sigma_{i} \sigma_{i},|i-j| \geq 2
\end{array}
$$


the inverse of $\sigma_{i}$ is $\sigma_{i}^{-1}$, the identity is denoted by $I$, and the centre of $B_{n}$ is generated by $\left(\sigma_{1} \sigma_{2} \cdots \sigma_{N-1}\right)^{N}$. The multivaluedness of the eigenfunction originates from the phase change in effecting an interchange between two coordinates $x_{i}^{(1)}$ and $x_{i}^{(2)}$ (superscripts refering to components) which can be expressed as

$$
\begin{array}{r}
V=\exp \left(i \nu \sum_{i<j} \phi_{i j}\right), \\
\phi_{i j}=\tan ^{-1}\left(\frac{x_{i}^{(2)}-x_{j}^{(2)}}{x_{i}^{(1)}-x_{j}^{(1)}}\right) .
\end{array}
$$

The description adopted by us here is referred to as the Anyon Gauge. It is important to realise that a set of coordinate configuration can be reached starting from some initial coordinates of $N$ particles in an infinite ways, each possibility manifested by an action of an element $\beta \in B_{N}$.

The connection between initial and final sequences is given by (3), via the character $\chi(\beta)$ of the specific element. Thus, to every $\beta \in B_{N}$, we can associate the affected partial amplitude $\psi_{\alpha}(\beta: \vec{x})$ [16. With one-dimensional unitary representation of the braid group, the rudiments of quantum mechanics allow us to write

$$
\Phi_{\alpha}(\vec{X})=\sum_{\beta \in B_{n}} \chi(\beta) \psi_{\alpha}(\beta: \vec{X})
$$

where $\psi_{\alpha}(\beta: \vec{X})$ is the probability amplitude associated in changing a configuration $\vec{X}$ to $(\beta: \vec{X})$ - a configuartion after the action of $\beta$ on $\vec{X}$. The wavefunction $\Phi_{\alpha}(\vec{X})$ is to be understood as appropriately normalised. The ansatz for $V \psi_{\alpha}(\beta: \vec{X})$ is now

$$
V \psi_{\alpha}(\beta: \vec{X})=N_{\alpha} \int d^{2 N} \vec{P} A_{\alpha}(\beta: \vec{P}) \delta\left(P^{2}-2 m E_{\alpha}\right) e^{\frac{i}{\hbar} \vec{X} \cdot \vec{P}}
$$

with $A_{\alpha}(\beta: \vec{P})$ satisfying

$$
\left\langle A_{\alpha}^{*}\left(\beta_{1}: \vec{P}_{1}\right) A_{\gamma}\left(\beta_{2}: \vec{P}_{2}\right)\right\rangle_{M E}=\delta_{\alpha \gamma} \frac{\delta^{2 N}\left(\left(\beta_{1}: \vec{P}_{1}\right)-\left(\beta_{2}: \vec{P}_{2}\right)\right)}{\delta\left(\vec{P}_{1}^{2}-\vec{P}_{2}^{2}\right)},
$$

$\left(\beta_{1}, \beta_{2} \in B_{N}\right)$, and $A_{\alpha}(\vec{P})$ satisfy the twisted boundary conditions,

$$
\begin{array}{r}
A_{\alpha}\left(\vec{p}_{1}, \cdots, \vec{p}_{i}, \cdots, \vec{p}_{j}, \cdots, \vec{p}_{N}\right) \\
=e^{i \pi \nu} A_{\alpha}\left(\vec{p}_{1}, \cdots, \vec{p}_{j}, \cdots, \vec{p}_{i}, \cdots, \vec{p}_{N}\right)
\end{array}
$$


The question now is in specifying exactly what the matrix ensemble is in this case? The form of (8) with $A_{\alpha}$ 's not restricted to real, takes into account the T-breaking, and (9) makes the ensemble handed or chiral as a result of P-breaking. Thus (7)-(9) gives the complete description and the ME is, in fact, the chiral-Gaussian Unitary Ensemble (ch-GUE) [17]. It can be easily shown that the Wigner distribution is

$$
\begin{aligned}
\left\langle\rho_{\alpha}^{W}(\vec{X}, \vec{P})\right\rangle_{M E} & =n_{\alpha}^{-1} h^{-2 N} \delta\left(\frac{P^{2}}{2 m}-E_{\alpha}\right), \\
n_{\alpha} & =\frac{1}{N ! \Gamma(N) E_{\alpha}}\left(\frac{m L^{2} E_{\alpha}}{2 \pi \hbar^{2}}\right)^{N}
\end{aligned}
$$

For the momentum distribution, we need to evaluate the ME-average of $\tilde{\Phi}_{\alpha}^{*}(\vec{P}) \tilde{\Phi}_{\gamma}^{*}\left(\vec{P}^{\prime \prime}\right)$ with $\tilde{\Phi} \equiv V \Phi_{\alpha}$. With the above ansatz and conditions supplementing it, this average is

$$
\begin{aligned}
& \mathcal{F}(\vec{P})=\left\langle\tilde{\Phi}_{\alpha^{\prime}}^{*}(\vec{P}) \tilde{\Phi}_{\gamma}^{*}\left(\vec{P}^{\prime \prime}\right)\right\rangle_{M E}= \\
& h^{2 N} \delta_{\alpha^{\prime} \gamma} N_{\alpha^{\prime}} N_{\gamma} \sum_{n, m=0} \sum_{\beta_{1}(m)} \sum_{\beta_{2}(n)} \chi^{*}\left(\beta_{1}\right) \chi\left(\beta_{2}\right) \delta\left(P^{2}-2 m E_{\alpha^{\prime}}\right) \\
& \times\left.\delta_{\mathcal{D}}^{2 N}\left(\prod_{\alpha=0}^{m} \sigma_{\beta_{1}(\alpha)}^{\epsilon_{\beta_{1}}} \vec{P}^{\prime \prime}-\prod_{\alpha=0}^{n} \sigma_{\beta_{2}(\alpha)}^{\epsilon_{\beta_{2}}} \vec{P}\right)\right|_{\vec{P}=\vec{P},}
\end{aligned}
$$

where

$$
\delta_{\mathcal{D}}^{2 N}(\vec{Q})=h^{-2 N} \int_{\text {Domain }, \mathcal{D}} d^{2 N} X \exp \left(\frac{i}{\hbar} \vec{Q} \cdot \vec{X}\right)
$$

$\vec{P}$ is identified with $\vec{P}^{\prime \prime}$ after the sum is performed.

With (11), the momentum distribution is given by

$$
F\left(\vec{p}_{1}\right)=\frac{\int d \vec{p}_{2} \cdots d \vec{p}_{N} \mathcal{F}(\vec{P})}{\int d \vec{p}_{1} \cdots d \vec{p}_{N} \mathcal{F}(\vec{P})}
$$

which formally completes the deduction. However, an exact evaluation of this is very difficult and the difficulty is coming from counting of irreducible words formed by the $\sigma^{\prime} s$. To make the precise connection, we give results upto $O\left(\hbar^{2} / L^{2}\right)$, an order that is enough for second virial coefficient. Leaving the tedium of details to a later publication, we just give our result incorporating all elements leading to $O\left(\hbar^{2} / L^{2}\right)$,

$$
\begin{aligned}
& F_{2}\left(\vec{p}_{1}\right)=(2 \pi m k T)^{-1} \exp \left(-\frac{\vec{p}_{1}^{2}}{2 m k T}\right)\{1 \\
& \left.+\left(\frac{h}{L}\right)^{2} \frac{1}{2 \pi m k T}\left(2 e^{-\frac{\vec{p}_{1}^{2}}{2 m k T}}-1\right) G(N, \nu)+O\left(\frac{h^{4}}{L^{4}}\right)\right\}
\end{aligned}
$$


where

$$
G(N, \nu)=\frac{\sum_{m=0}^{\infty} \sum_{K=-2 m-1(\text { even })}^{2 m+1} Q_{K}^{(m)}(N) \cos (\pi K \nu)}{1+2 \sum_{m=1}^{\infty} \sum_{K=-2 m(\text { odd })}^{2 m} P_{K}^{(m)}(N) \cos (\pi K \nu)}
$$

$Q_{K}^{(m)}$ is the number of elements in $B_{N}$ composed of ' $m$ ' generators whereby the momentum $\vec{p}_{1}$ is interchanged with another momentum yielding a character $\exp (i \pi K \nu)$ (or $\exp (-i \pi K \nu)$ since $\left.Q_{K}^{(m)}(N)=Q_{-K}^{(m)}(N)\right) ; P_{K}^{(m)}(N)$ is the number of elements in $B_{N}$ contributing to identity with a character $\exp (i \pi K \nu)($ or $\exp (-i \pi K \nu))$. Temperature is introduced above via the ideal gas law, $E_{\alpha}=N k T_{\alpha}$. Unfortunately though, this counting problem stands open today [18]. It is very important to note that the ansatz (7)-(9) for the special case when $\sigma_{i}^{2}=1$ for all $i$ where $B_{N}$ reduces to symmetric group, $S_{N}$, the well-known Fermi-Dirac and Bose-Einstein distributions follow. On evaluating pressure, $\Pi$ from (14), denoting area of the enclosure by $\mathrm{A}$, we get $\Pi A / k T=1-(2 A)^{-1} \lambda^{2} G(N, \nu)$, with $\lambda^{2}=h^{2}(2 \pi m k T)^{-1}$. We immediately see that $G(N, 0) /(2 N)$ and $G(N, 1) /(2 N)$ are $2^{-3 / 2}$ and $-2^{-3 / 2}$ respectively yielding the second virial coeffiecient for the Bose and Fermi gases [19]. For the fractional case, with $\nu=$ even number, $2 \mathrm{j}+\delta$ ("boson-based anyons"), comparing our result with [8], we get the Sum Rule mentioned in the introduction:

$$
-2^{-3 / 2} N^{-1} G(N, \nu) \lambda^{2}=\left(-1+4|\delta|-2 \delta^{2}\right) \lambda^{2} / 4
$$

the right hand side belongs to $[8]$. It is important to note that our deduction is nonperturbative and in principle, we can get expressions for higher-order virial coefficients also [20]. To understand this, we observe that the relation (8) connects two momentum configurations of $\mathrm{N}$ particles, and not just the momenta of two particles. Thus, it contains information that can lead to all virial coefficients. For example, for the third virial coefficient, we need to evaluate contributions to $F\left(\vec{p}_{1}\right)$ when three momenta out of $\mathrm{N}$ are interchanged. The denominator of (13) contains those interchanges which braid three strands in such a way that the initial configuration of momenta is preserved whereas the numerator of (13) contains those which exchange the momentum assignment on all three strands. We have done the calculation and the third virial coefficient is expressible in terms of the specific counting 
problem of $B_{N}$. Here, in order to convince the reader, it suffices to make a comparative discussion with the existing calculation. For this, we write down the total contribution to the momentum distribution due to a triple interchange emerging from the elements of $B_{N}$ formed by M generators,

$$
\begin{aligned}
\left(\frac{L}{\hbar}\right)^{2(N-2)} & \quad \sum_{-2 M}^{2 M} \frac{2}{3} I_{2(N-3)}\left(2 m E_{\alpha}-\vec{p}_{1}^{2}\right) \cos (\pi k \nu) \mathcal{R}_{k}^{M}(N) \\
+ & 2 I_{2(N-3)}\left(2 m E_{\alpha}-3 \vec{p}_{1}^{2}\right) \cos (\pi k \nu) \mathcal{S}_{k}^{M}(N)
\end{aligned}
$$

where $\mathcal{S}_{k}^{M}(N)\left(\mathcal{R}_{k}^{M}(N)\right)$ are the number of elements of $B_{N}$ that (do not) change the momentum $\vec{p}_{1} . I_{D}(x)$ denotes the volume of a D-dimensional hyper-sphere of radius $\mathrm{x}$. The reason we give this result here is to show that (17) is a Fourier series with harmonic terms like $\cos 2 \pi \nu, \cos 4 \pi \nu$, and so on, in complete agreement with the conjectured form [21. It is becoming evident from the Monte Carlo calculations [22] that the third virial coefficient is a series with terms as $\sin ^{2} \pi \nu, \sin ^{4} \pi \nu$, and so on. Our formal result is thus in consonance with these works. Also, we mention that (16), (17) and the Monte Carlo estimates provide a non-trivial hint on the counting problem itself.

To summarise, we have formulated in this Letter a way to approach Statistical Mechanics from RMT through the ansatz for eigenfunctions which is an essential dynamical input. Moreover, we have presented a first-principles-evaluation of the momentum distribution of a 'Hard-Sphere Anyon Gas' in the low-density regime which is of a great interest in current literature. The nature of difficulties, albeit well-known, are made explicit here in direct connection with the existing wisdom (or ignorance) of theory of braid groups. The parameter, $\nu$ has an analogous partner in quantum chromodynamics [8] and we conjecture that the anyon gas discussed here and the $\nu \pi$-parametrised quantum chromodynamics belong to the same universality class of chiral-Gaussian Unitary Ensemble of RMT. 


\section{ACKNOWLEDGEMENTS}

Authors express their deep debt of gratitude to Pierre Gaspard for stimulating discussions

and constructive criticism. S.R.J. is financially supported by the "Communaute Francaise de Belgique" under contract no. ARC-93/98-166. 


\section{REFERENCES}

[1] O. Bohigas, M.-J. Giannoni and C. Schmit, Phys. Rev. Lett., 52, 1(1984). S.W. McDonald, Ph.D. Thesis (UMI 8413506, Ann Arbor, MI, 1983).

[2] S.W. McDonald and A.N. Kaufmann, Phys. Rev. A 37, 3067 (1988); D. Biswas and S. R. Jain, Phys. Rev. A 42, 3170 (1990); V.N. Prigodin. N. Taniguchi, A. Kudrolli, V. Kidambi, S. Sridhar, "Spatial Correlation in Quantum Chaotic Systems with TimeReversal Symmetry: Theory and Experiment", preprint (1995); M. Srednicki, Comment on "Spatial.." by Progodin et al. preprint (1995).

[3] M. V. Berry, J. Phys. A10, 2083(1977).

[4] M. Srednicki, Phys. Rev. E 50, 888 (1994).

[5] A. Lerda, 'Anyons', Lecture Notes in Physics (m-14) (Springer, N. Y., 1992).

[6] R.E. Prange and S. M. Girvin, Eds. "The Quantum Hall Effect" (Springer, N. Y., 1990); R. Ladbury, Phys. Today, July 1995, p. 19.

[7] J. M. Leinaas and J. Myrheim, Nuovo Cim. 37B, 1(1977); F. Wilczek, Phys. Rev. Lett. 48, 114(1982); 49, 957(1982).

[8] D. Arovas, R. Schrieffer, F. Wilczek and A. Zee, Nucl. Phys. B251, 117(1985).

[9] E. C. G. Stueckelberg, Helv. Phys. Acta 32, 254(1959); 33, 727(1960).

[10] F. J. Dyson, J. Math. Phys. 3, 1199(1962).

[11] J. Fröhlich and P. Marchetti, Comm. Math. Phys. 121, 177(1989).

[12] G. Frobenius, J. reine u. angew. Math. 84, 59 (1878).

[13] C. E. Porter, Statistical Theories of Spectra : Fluctuations, (Academic Press, New York, 1965), p. 64ff.

[14] L. van Hove, Physica 21, 517(1955); 25, 268(1959). 
[15] E. Artin, Ann. Math. 48, 101(1947); F. Bohnenblust, Ann. Math. 48, 127(1947).

[16] M. G. G. Laidlaw and C. DeWitt-Morette, Phys. Rev. D3, 1375(1971); Y.-S. Wu, Phys. Rev. Lett. 52, 2103(1984).

[17] This terminology is used in recent literature, see e.g. J. Verbaarschot, Phys. Rev. Lett. 72, 2531(1994); we have not examined a relation between our definition and the sense in which it is used in this work. We are grateful to Pierre Gaspard for bringing this work to our attention.

[18] J. S. Birman and A. Libgoher, Eds. Contemporary Mathematics : Braids, Proc. Summer Research Conference, July 13-26, 1986, Volume 78 (American Mathematical Society), see particularly pp. 573-574.

[19] K. Huang, Statistical Mechanics (John Wiley and Sons Inc., New York, 1987).

[20] D. Alonso and S. R. Jain, in preparation.

[21] J. Myrheim and K. Olaussen, Phys. Lett. B299, 267 (1993).

[22] S. Mashkevich, J. Myrheim, and K. Olaussen, preprint (1996). 